\title{
Predictive value and changes of miR-34a after concurrent chemoradiotherapy and its association with cognitive function in patients with nasopharyngeal carcinoma
}

\author{
WEIYU DENG ${ }^{1}$, ANQI LIN ${ }^{2}$ and JIALIN YANG ${ }^{3}$ \\ Departments of ${ }^{1}$ Neurology, ${ }^{2}$ Pneumology and ${ }^{3}$ Oncology, Shunde Hospital, Guangzhou \\ University of Traditional Chinese Medicine, Foshan, Guangdong 528300, P.R. China
}

Received July 1, 2019; Accepted May 27, 2020

DOI: $10.3892 / \mathrm{ol} .2020 .11995$

\begin{abstract}
This study explored the changes and predictive value of miR-34a in nasopharyngeal carcinoma (NPC) after concurrent chemoradiotherapy (CCRT), and its association with cognitive function. Fifty NPC patients admitted to Shunde Hospital, and another fifty healthy individuals were assigned into treated group and control group, respectively. Patients in the treated group received 3 courses of CCRT. The relative expression of miR-34a in the two groups was detected, and the cognitive function of patients was assessed. Diagnostic and predictive values of miR-34a in advanced NPC were analyzed. The expression of miR-34a in the control group was significantly higher than that in the treated group $(\mathrm{t}=13.364, \mathrm{P}<0.001)$, with an area under the curve (AUC) of 0.979 . The expression of miR-34a was significantly upregulated after treatment $(\mathrm{t}=4.559, \mathrm{P}<0.001)$. After treatment, there were 32 complete remission (CR) patients and 18 partial remission (PR) patients. According to efficacy, CR patients were classified as significant group and PR patients as general group. The expression of miR-34a in the significant group was higher than that in the general group before treatment $(\mathrm{t}=4.704, \mathrm{P}<0.001)$, with an AUC of 0.852 . The Montreal Cognitive Assessment (MoCA) score was significantly decreased after treatment $(\mathrm{t}=13.042, \mathrm{P}<0.001)$. The expression of miR-34a was positively correlated with the MoCA score after treatment, that is, MoCA score gradually increased with the upregulation of miR-34a expression $(r=0.379, \mathrm{P}=0.006)$. There is a positive correlation between miR-34a and cognitive function of patients. Moreover, the expression of miR-34a can be used as a potential predictor of the efficacy of CCRT in patients with NPC.
\end{abstract}

Correspondence to: Dr Jialin Yang, Department of Oncology, Shunde Hospital, Guangzhou University of Traditional Chinese Medicine, Daliang Jinsha Avenue, Foshan, Guangdong 528300, P.R. China

E-mail:.jpxr71@163.com

Key words: miR-34a, nasopharyngeal carcinoma, cognitive function

\section{Introduction}

Nasopharyngeal carcinoma (NPC) is a highly malignant tumor arising from epithelial cells (1). In 2015, there were 60,600 newly diagnosed NPC and 34,100 deaths in China (2). Despite the low morbidity and mortality, the treatment of NPC has always been difficult in clinical practice. NPC is occult and adjacent to optic nerve, trigeminal nerve, and internal carotid artery, so it is difficult to be treated by surgery (3). With the exception of a small number of patients with early NPC who can be cured by radical surgery, most patients are treated with palliative chemoradiotherapy to improve the condition (4). At present, there are few efficacy assessment indicators for NPC, and imaging examinations are mainly applied to assess the efficacy of chemoradiotherapy (5). However, serological test is less harmful, faster and more convenient. Therefore, it is of great significance to find a specific serological index (6).

Patients with NPC always suffer from cognitive decline after chemoradiotherapy. Currently, Montreal Cognitive Assessment (MoCA) score and Das-Naglieri cognitive assessment system are commonly used to assess the cognitive function of patients (7). However, subjective bias may occur in artificial scoring, so it is very important to find a new observation index for cognitive function assessment (8). MicroRNAs (miRs) is a class of newly discovered, highly conserved, endogenous, non-coding, hairpin nucleotide transcripts that widely exist in eukaryotic cells and are approximately 19-25 bases in length and 18-25 nucleotides in size (9). Some miRNAs are reported to be closely related to the occurrence and progression of NPC (10). As an important member of the miR-34 family, miR-34a is located at 1p36.23 and mainly distributed in brain tissue under normal circumstances. A study found that it has a significantly reduced expression in NPC and was expected to become a potential diagnostic indicator for NPC (11). Another study suggested that miR-34a was closely associated to cognitive function, that is, cognitive function could be improved by downregulating the expression of miR-34a (12). Therefore, we speculate that there may be a correlation between miR-34a and cognitive function, but whether miR-34a is related to the cognitive impairment in patients with NPC has not been confirmed yet.

Therefore, this study explored the potential value of miR-34a in assessing cognitive function and radiotherapy 
efficacy in patients with NPC by determining its expression before and after radiotherapy, so as to provide reference for clinicians.

\section{Patients and methods}

Clinical data. A total of 89 patients with NPC admitted to Shunde Hospital (Foshan, China) from July 2015 to February 2017 were enrolled, 39 patients who failed to complete treatment were excluded, and the remaining 50 served as a treated group. Moreover, 50 healthy individuals who underwent physical examination in the hospital during the same period were collected as a control group. Laboratory test indexes and imaging tests of patients in the control group were normal. The study was approved by the Ethics Committee of Shunde Hospital. Signed informed consents were obtained from the patients and/or guardians. Inclusion criteria: Patients aged over 18 years; patients conforming to AJCC 7th version (13) and staged III-IVB; patients diagnosed with NPC through imaging and pathological examinations; Eastern Cooperative Oncology Group (ECOG) score $\leq 1$. Exclusion criteria: Patients complicated with other tumors; patients receiving corresponding anti-cancer treatment before this treatment; patients intolerant to chemoradiotherapy; breast-feeding women and patients with cognitive dysfunction.

Main reagents and drugs. Cisplatin sodium chloride injection (Guizhou Hanfang Pharmaceutical Co., Ltd.); total RNA extraction kit EasyPure miRNA kit - TransScript Green miRNA Two-Step qRT-PCR SuperMix (Beijing TransGen Biotech Company; AQ 202-01, ER601-01); PCR instrument (ABI 7500; Applied Biosystems; Thermo Fisher Scientific, Inc.).

Treatment. All the patients were treated with concurrent chemoradiotherapy (CCRT), and $6 \mathrm{MV}-\mathrm{X}$-ray, 9-field three-dimensional conformal radiotherapy (3D-CRT) and intensity modulated radiotherapy (IMRT) were performed. The specific steps were as follows: The tumor volume was measured by MRI and CT localization images, prescription dose: PGTVnx, 70.4-72.3 Gy/32 f; PGTVnd, 66-70.4 Gy/32 f; PCTV 1, 60-62 Gy/30 f; PCTV 2, 54-55.8 Gy/30 f, once a day, five times a week. The radiation dose to temporal lobes was obtained using dose-volume histogram (DVH). Chemotherapy was performed with cisplatin at the same time. The specific protocol was as follows: One cycle of cisplatin $\left(100 \mathrm{mg} / \mathrm{m}^{2}\right)$ was given on the 1st, 22nd and 43rd days, respectively, 21 days each cycle (14).

Expression of miR-34a in serum of patients. Samples of peripheral blood $(5 \mathrm{ml})$ were collected from patients before and after 3 cycles of CCRT (after treatment) and from subjects in the control group, placed at room temperature for $30 \mathrm{~min}$, and centrifuged at $1,500 \mathrm{x}$ g for $10 \mathrm{~min}$ to collect the serum. Total RNAs were extracted using a EasyPure miRNA kit, and the purity, concentration and integrity were detected using an ultraviolet spectrophotometer and agarose gel electrophoresis. The RNAs were reverse transcribed into cDNAs by $2 \mathrm{X}$ TS miRNA reaction mix in transScript Green miRNA two-step qRT-PCR supermix kit, and the specific procedure was performed according to the manufacturer's kit instructions. Then PCR
Table I. Efficacy evaluation.

\begin{tabular}{ll}
\hline Efficacy grade & \multicolumn{1}{c}{ Evaluation criteria } \\
\hline $\mathrm{CR}$ & $\begin{array}{l}\text { After treatment, the lesions disappeared } \\
\text { completely for }>4 \text { weeks }\end{array}$ \\
$\mathrm{PR}$ & $\begin{array}{l}\text { After treatment, the total maximum diameter } \\
\text { of the lesions decreased by } \geq 50 \%\end{array}$ \\
$\mathrm{SD}$ & $\begin{array}{l}\text { After treatment, the total maximum diameter } \\
\text { of the lesions decreased by }<50 \%\end{array}$ \\
$\mathrm{PD}$ & $\begin{array}{l}\text { After treatment, the total maximum diameter } \\
\text { of the lesions decreased by } \geq 25 \% \text { or new } \\
\text { lesions appeared }\end{array}$ \\
\hline
\end{tabular}

$\mathrm{CR}$, complete remission; $\mathrm{PR}$, partial remission; $\mathrm{SD}$, stable disease; $\mathrm{PD}$, progressive disease.

amplification was carried out. PCR reaction system: $1 \mu \mathrm{l}$ of cDNA, $0.4 \mu \mathrm{l}$ of each upstream and downstream primers, $10 \mu \mathrm{l}$ of 2X TransTaq ${ }^{\circledR}$ Tip Green qPCR SuperMix, $0.4 \mu 1$ of Passive Reference Dye (50X), finally made up to $20 \mu \mathrm{l}$ with $\mathrm{ddH}_{2} \mathrm{O}$. PCR reaction conditions: Pre-denaturation at $94^{\circ} \mathrm{C}$ for $30 \mathrm{sec}$, denaturation at $94^{\circ} \mathrm{C}$ for $5 \mathrm{sec}$, annealing at $60^{\circ} \mathrm{C}$ for $15 \mathrm{sec}$, extension for $10 \mathrm{sec}$, for a total of 40 cycles. Each sample was tested in 3 repeat wells, and the experiment was carried out 3 times. In this study, U6 was used as an internal reference and the $2^{-\Delta \Delta C q}$ was used to analyze the data (15). miR-34a upstream primer 5'-GTGCAGGGTCCGAGGT-3', downstream primer 5'-GCCGCTGGCAGTGTCTTAGCTG-3', U6 upstream primer 5'-CTCGCTTCGGCAGCACA-3', downstream primer 5'-AACGCTTCACGAATTTGCGT-3'.

Efficacy evaluation criteria. According to Response Evaluation Criteria in Solid Tumors, the short-term efficacy was evaluated and divided into 4 grades: Complete remission (CR), partial remission (PR), stable disease (SD) and progressive disease (PD (Table I).

Outcome measures. Main outcome measures: The expression of miR-34a in the control and the treated group before treatment was compared. The diagnostic value of miR-34a was assessed using a receiver operating characteristic (ROC) curve, and the changes of miR-34a before and after treatment were compared. The changes of MoCA score (all the subjects were scored by a senior psychiatrist in the hospital) before and after treatment were compared. A higher score indicated a better cognitive function of the patients (30 points in total).

Secondary outcome measures: CR patients were classified as significant group and PR patients as general group. The expression of miR-34a before treatment was compared between the two groups, and the area under the ROC curve (AUC) was obtained. The correlation between miR-34a and MoCA score after treatment was analyzed.

Statistical analysis. In this study, SPSS 20.0 software package (Guangzhou Pomine Information Technology Co., Ltd.) was used to carry out statistical analysis on the collected 


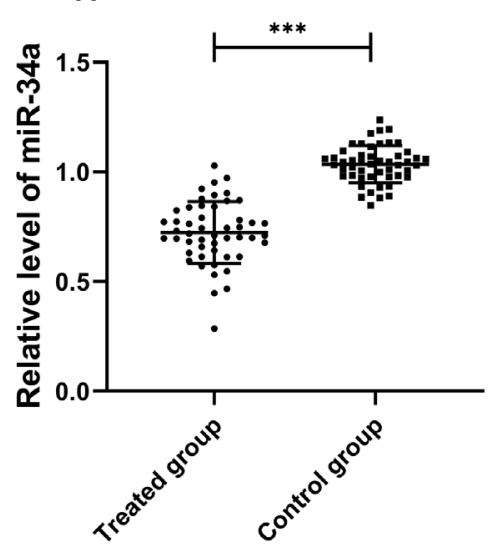

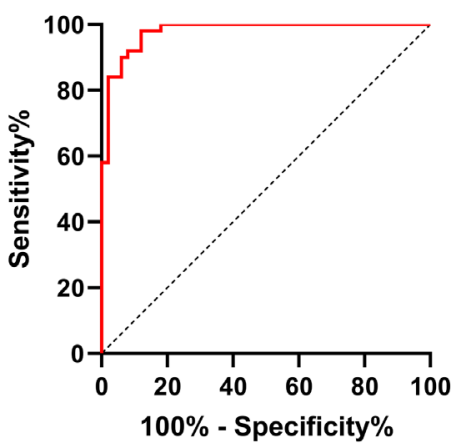

Figure 1. Expression and diagnostic value of miR-34a in NPC. (A) Comparison of serum miR-34a expression in normal individuals and in NPC patients. (B) Diagnostic value of miR-34a in the diagnosis of advanced NPC. When cut-off was 0.879 , the optimal specificity, sensitivity and Youden index were 88.00 , 98.00 and $86.00 \%$, respectively. ${ }^{* * *} \mathrm{P}<0.001$. miR, microRNA; NPC, nasopharyngeal carcinoma.

Table II. Comparison of baseline data.

\begin{tabular}{|c|c|c|c|c|}
\hline Factor & $\begin{array}{l}\text { Observation } \\
\text { group } \\
(n=50)\end{array}$ & $\begin{array}{l}\text { Control } \\
\text { group } \\
(n=50)\end{array}$ & $t / \chi^{2}$ & P-value \\
\hline \multicolumn{5}{|l|}{ Sex } \\
\hline Male & $39(78.00)$ & $42(84.00)$ & 0.585 & 0.444 \\
\hline Female & $11(22.00)$ & $8(16.00)$ & & \\
\hline Age (years) & $52.1 \pm 7.2$ & $53.4 \pm 6.3$ & 0.961 & 0.339 \\
\hline BMI $\left(\mathrm{kg} / \mathrm{m}^{2}\right)$ & $21.25 \pm 1.47$ & $21.48 \pm 1.55$ & 0.761 & 0.448 \\
\hline \multicolumn{5}{|l|}{ Clinical staging } \\
\hline III & $5(10.00)$ & & & \\
\hline IVa & $36(72.00)$ & & & \\
\hline $\mathrm{IVb}$ & $9(18.00)$ & & & \\
\hline \multicolumn{5}{|l|}{$\mathrm{T}$ staging } \\
\hline $\mathrm{T} 2$ & $3(6.00)$ & & & \\
\hline $\mathrm{T} 3$ & $4 \quad(8.00)$ & & & \\
\hline $\mathrm{T} 4$ & $43(86.00)$ & & & \\
\hline \multicolumn{5}{|l|}{$\mathrm{N}$ staging } \\
\hline N0 & $4 \quad(8.00)$ & & & \\
\hline N1 & $8(16.00)$ & & & \\
\hline N2 & $27(54.00)$ & & & \\
\hline N3 & $11(22.00)$ & & & \\
\hline \multicolumn{5}{|l|}{$\begin{array}{l}\text { Pathological } \\
\text { type }\end{array}$} \\
\hline $\begin{array}{l}\text { Keratinizing } \\
\text { squamous cell } \\
\text { carcinoma }\end{array}$ & $25(50.00)$ & & & \\
\hline $\begin{array}{l}\text { Non-keratinizing } \\
\text { squamous cell } \\
\text { carcinoma }\end{array}$ & $25(50.00)$ & & & \\
\hline
\end{tabular}

data. GraphPad Prism 7 (Shanghai Cabit Information Technology Co., Ltd.) was used to draw the figures. Kolmogorov-Smirnov (K-S) test was used to analyze the data distribution. Counting data expressed as percentage (\%)

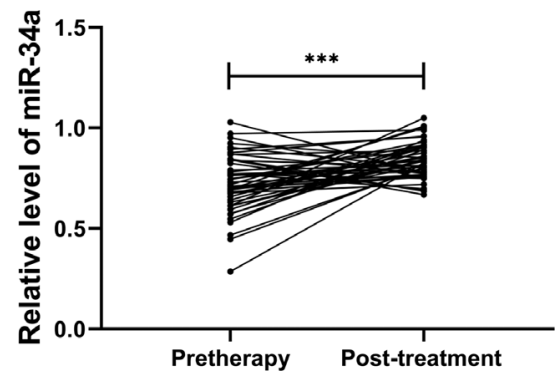

Figure 2. Expression of miR-34a in the treated group before and after treatment. ${ }^{* * * *} \mathrm{P}<0.001$. miR, microRNA.

were analyzed using the Chi-square test (denoted by $\chi^{2}$ ). Measurement data conforming to normal distribution and variance homogeneity were expressed as the mean \pm SD. Independent t-test was used for analysis between the two groups, while paired t-test was used for comparison before and after treatment within the group (denoted by $\mathrm{t}$ ). ROC was used to assess the predictive and diagnostic values of miR-34a for NPC. Pearson test was used for determining the correlation between miR-34a and MoCA score after treatment. $\mathrm{P}<0.05$ indicates a statistically significant difference.

\section{Results}

Comparison of baseline data. There was no significant difference in sex, age and body mass index (BMI) $(\mathrm{P}>0.05)$ between the two groups, as shown in Table II.

Expression and diagnostic value of miR-34a in NPC. The expression of miR-34a in the control group (1.035 \pm 0.085$)$ was significantly higher than that in the treated group $(0.723 \pm 0.141)$ $(\mathrm{t}=13.364, \mathrm{P}<0.001)$. ROC curve showed that the AUC of miR-34 was 0.979, showing a high diagnostic value (Fig. 1).

miR-34a expression before and after treatment. The expression of miR-34a in the treated group increased significantly after treatment compared with that before treatment $(\mathrm{t}=4.559$, $\mathrm{P}<0.001$ ), as shown in Fig. 2. 
A

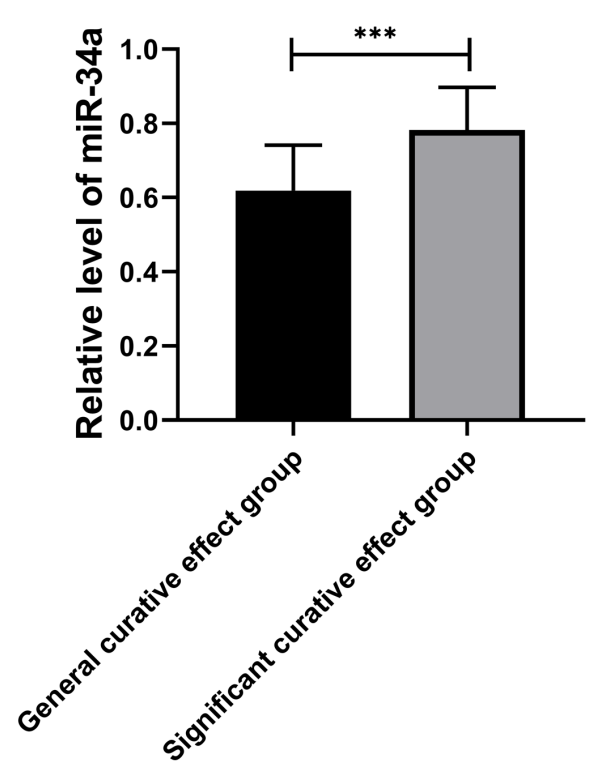

B

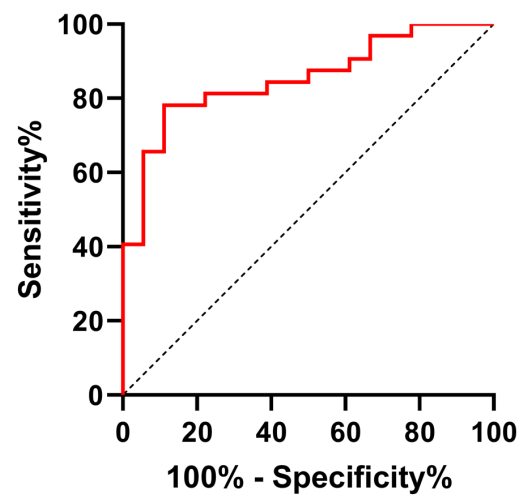

Figure 3. Predictive value of miR-34a in NPC. (A) Expression of miR-34a in significant group and general group before treatment. (B) When cut-off was 0.706, the optimal specificity, sensitivity and Youden index were $88.89,78.13$ and $67.01 \%$, respectively. ${ }^{* * *} \mathrm{P}<0.001$, miR, microRNA; NPC, nasopharyngeal carcinoma.

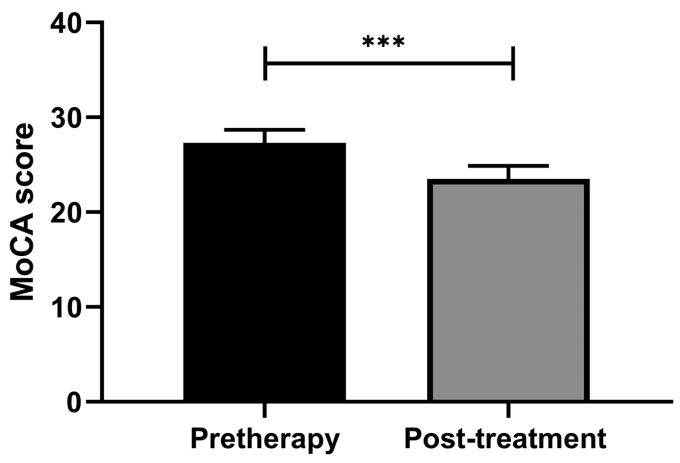

Figure 4. MoCA score changes before and after treatment in treated group. ${ }_{* * * *} \mathrm{P}<0.001$. MoCA, Montreal Cognitive Assessment.

Predictive value of miR-34a in NPC. Evaluation of the clinical efficacy of patients in the treated group showed that there were $32 \mathrm{CR}$ patients and $18 \mathrm{PR}$ patients after treatment. The CR patients were classified as the significant group and PR patients as the general group. The expression of miR-34a in the significant group was higher than that in the general group before treatment $(\mathrm{t}=4.704, \mathrm{P}<0.001)$. The ROC curve showed that the AUC was 0.852 (Fig. 3).

MoCA score changes before and after treatment. The MoCA score of patients after treatment $(23.52 \pm 1.37)$ was significantly lower than that before treatment $(27.32 \pm 1.36)(\mathrm{t}=13.042$, $\mathrm{P}<0.001$ ) (Fig. 4).

Correlation between miR-34a expression and MoCA score after treatment. Pearson correlation analysis showed that the expression of miR-34a was positively correlated with the MoCA score after treatment, that is, MoCA score increased gradually with the increase of miR-34a expression $(r=0.379$, $\mathrm{P}=0.006$ ), as shown in Fig. 5 .

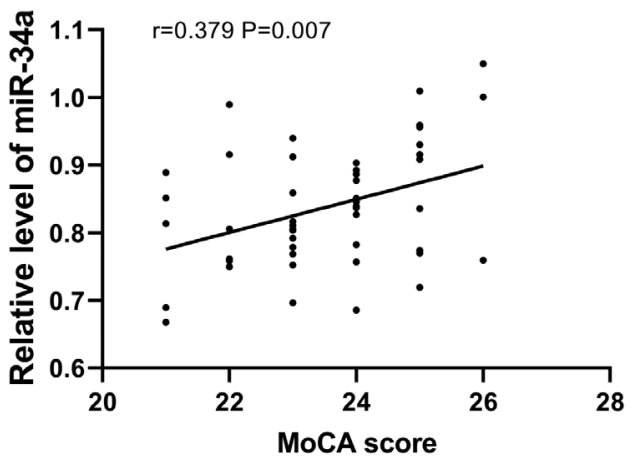

Figure 5. Correlation between miR-34a expression and MoCA score after treatment. miR, microRNA; MoCA, Montreal Cognitive Assessment.

\section{Discussion}

Due to the concealed onset of NPC, majority of patients are in advanced stage when diagnosed, missing the optimal treatment timing. At present, early NPC patients are mainly treated with radiotherapy alone, and patients in advanced stage are treated with platinum plus radiotherapy $(16,17)$. Efficacy evaluation is mainly based on imaging tests after treatment that determine the size of solid tumors. However, the tests have a certain impact on the human body, and the imaging results take time (18). Therefore, we questioned whether it is possible to predict the efficacy by performing tests on the patient before treatment, in order to assess the clinical effect in advance and to improve the treatment efficiency. However, there are few relevant indicators for clinical prediction of clinical efficacy, so it is very important to find one.

miRs are a class of highly conserved non-coding small RNAs widely distributed in eukaryotic cells, which play an important role in the development and progression of malignant tumors (19). Studies have shown that $(20,21)$ a 
variety of miRs are capable of regulating the occurrence and development of NPC. Previous studies have found that miR-34a, a member of the miRs family, regulates biological function in prostate cancer and renal cell cancer $(22,23)$. Recent studies have shown that miR-34a has a low expression in NPC (24). However, whether it can be used as a efficacy predictor has not been studied. Therefore, in this study, the predictive value of miR-34a in efficacy evaluation of NPC patients after CCRT was explored to provide indicators for the clinic. The expression of serum miR-34a was first detected, and the results showed that the expression in the treated group was significantly lower than that in the control group. Moreover, the ROC curve showed that miR-34a had extremely high diagnostic value in patients with advanced NPC. In the study of Long et al (25), it was found that hyperfration radiotherapy enhanced cell apoptosis and upregulated miR-34a expression. Moreover, the expression of miR-34a before and after treatment through clinical trials was compared, and it was found that the expression was significantly increased after treatment, which was consistent with the results of Long et al (25), indicating that the expression of miR-34a increased after CCRT. Furthermore, the patients were divided into the significant group and the general group according to the curative effect, the expression of miR-34a before treatment between the two groups was compared. The results indicated that the expression in significant group was significantly higher than that in general group. ROC curve showed that the AUC was $>0.8$, suggesting that observing the expression of miR-34a before treatment was a potential predictor for assessing the efficacy of CCRT in patients with NPC. The higher the expression of miR-34a before treatment, the more significant improvement of the clinical curative effect.

Although the condition of the patient and survival are improved, the cognitive function of patients may be declined after radiotherapy, which reduces the quality of life of patients. At present, various scores and scale are preferred means to assess cognitive function, subjective errors are inevitable. A study (26) found that upregulation of miR-34a alleviated total abdominal irradiation (TAI)-mediated cognitive impairment by restoring BFNF expression in hippocampus. Therefore, we speculated whether the expression of miR-34a is related to the cognitive function of patients. Therefore, the changes of cognitive function of patients before and after treatment were compared, and it was found that the MoCA score after treatment was significantly lower than that before treatment. Qiu et al (27) stated that the MoCA score of NPC patients after radiotherapy was significantly lower than that before treatment, which was consistent with our research results. Moreover, Pearson correlation analysis showed that there was a positive correlation between miR-34a and MoCA score, indicating that the expression of miR-34a can be used as a potential observation indicator for patients with cognitive dysfunction. However, MoCA score may be affected by cancer staging, side effects, nutritional status and other factors, interfering with our findings. Thus, we will supplement more cognitive function related scales to investigate the association between miR-34a and cognitive function.

This study found that miR-34a can be used as a predictor of clinical efficacy and an observation indicator of cognitive function in NPC patients after CCRT. However, there are still limitations. The survival of NPC patients was not followed up, so whether miR-34a is related to the survival remains unknown. This is a clinical experiment, the relevant mechanism between miR-34a and patients' cognitive function is unclear. Only samples of patients with advanced NPC were collected, the expression of miR-34a in patients with early NPC and those without CCRT is not clear.

In conclusion, there is a positive correlation between miR-34a and cognitive function of patients. Moreover, observing the expression of miR-34a before treatment can be used as a potential observation predictor of the efficacy of CCRT in patients with NPC.

\section{Acknowledgements}

Not applicable.

\section{Funding}

No funding was received.

\section{Availability of data and materials}

The datasets used and/or analyzed during the present study are available from the corresponding author on reasonable request.

\section{Authors' contributions}

WD conceived and designed the study, and drafted the manuscript. WD, AL and JY collected, analyzed and interpreted the experimental data, and revised the manuscript critically for important intellectual content. All authors read and approved the final manuscript.

\section{Ethics approval and consent to participate}

The study was approved by the Ethics Committee of Shunde Hospital (Foshan, China). Signed informed consents were obtained from the patients and/or guardians.

\section{Patient consent for publication}

Not applicable.

\section{Competing interests}

The authors declare that they have no competing interests.

\section{References}

1. Chua MLK, Wee JTS, Hui EP and Chan ATC: Nasopharyngeal carcinoma. Lancet 387: 1012-1024, 2016.

2. Chen W, Zheng R, Baade PD, Zhang S, Zeng H, Bray F, Jemal A, Yu XQ and He J: Cancer statistics in China, 2015. CA Cancer J Clin 66: 115-132, 2016.

3. Sun Y, Li WF, Chen NY, Zhang N, Hu GQ, Xie FY, Sun Y, Chen XZ, Li JG, Zhu XD, et al: Induction chemotherapy plus concurrent chemoradiotherapy versus concurrent chemoradiotherapy alone in locoregionally advanced nasopharyngeal carcinoma: A phase 3, multicentre, randomised controlled trial. Lancet Oncol 17: 1509-1520, 2016. 
4. Ribassin-Majed L, Marguet S, Lee AWM, Ng WT, Ma J, Chan ATC, Huang PY, Zhu G, Chua DTT, Chen Y, et al: What is the best treatment of locally advanced nasopharyngeal carcinoma? An individual patient data network meta-analysis. J Clin Oncol 35: 498-505, 2017.

5. Chen YZ, Li WF, Wang JY, Wang JM, Ou RY, Zheng XW, Xu YS and Zhao L: Evaluation of time-phase effect on ${ }^{18} \mathrm{~F}$-FDG PET/CT delineation methods for treatment planning of nasopharyngeal carcinoma. Clin Nucl Med 41: 354-361, 2016

6. Chen Z, Xu L, Xu X and Yuan C: The clinical value of detecting circulating tumour cells in the peripheral blood of nasopharyngeal carcinoma patients. Oncol Lett 15: 6283-6290, 2018.

7. McDowell LJ, Ringash J, Xu W, Chan B, Lu L, Waldron J, Rock K, So N, Huang SH, Giuliani M, et al: A cross sectional study in cognitive and neurobehavioral impairment in long-term nasopharyngeal cancer survivors treated with intensity-modulated radiotherapy. Radiother Oncol 131: 179-185, 2019.

8. Fasfous AF, Al-Joudi HF, Puente AE and Pérez-García M: Neuropsychological measures in the Arab World: A systematic review. Neuropsychol Rev 27: 158-173, 2017.

9. Kozomara A, Birgaoanu M and Griffiths-Jones S: miRBase: From microRNA sequences to function. Nucleic Acids Res 47D: D155-D162, 2019.

10. Gao W, Lam JW, Li JZ, Chen SQ, Tsang RK, Chan JY and Wong TS: MicroRNA-138-5p controls sensitivity of nasopharyngeal carcinoma to radiation by targeting EIF4EBP1. Oncol Rep 37: 913-920, 2017.

11. Wu R, Qiu E, Lin R, Wang J and Lin H: Regulation of nasopharyngeal carcinoma cell proliferation by targeting Notch1 with miR-34a. Int J Clin Exp Pathol 9: 8811-8816, 2016.

12. Xu Y, Chen P, Wang X, Yao J and Zhuang S: miR-34a deficiency in APP/PS1 mice promotes cognitive function by increasing synaptic plasticity via AMPA and NMDA receptors. Neurosci Lett 670: 94-104, 2018.

13. Edge SB and Compton CC: The American Joint Committee on Cancer: the 7th edition of the AJCC cancer staging manual and the future of TNM. Ann Surg Oncol 17: 1471-1474, 2010.

14. Dechaphunkul A, Danchaivijitr P, Jiratrachu R, Dechaphunkul T, Sookthon C, Jiarpinitnun C, Paoin C, Setakornnukul J, Niyomnaitham S, Suktitipat B, et al: 1076P Comparison of 3 -weekly cisplatin versus 3-weekly carboplatin in patients with locally advanced nasopharyngeal carcinoma (LA-NPC) receiving concurrent chemoradiotherapy (CCRT): A multicenter retrospective study. Ann Oncol 29 (Suppl. 8): viii384, 2018

15. Livak KJ and Schmittgen TD: Analysis of relative gene expression data using real-time quantitative PCR and the 2(-Delta Delta C(T)) method. Methods 25: 402-408, 2001.

16. Ai QY, King AD, Mo FKF, Law BKH, Bhatia KS, Ma BB, Poon DMC and Kam MKM: Prediction of distant metastases from nasopharyngeal carcinoma: Improved diagnostic performance of MRI using nodal volume in N1 and N2 stage disease. Oral Oncol 69: 74-79, 2017.
17. Tan T, Lim WT, Fong KW, Cheah SL, Soong YL, Ang MK, Ng QS Tan D, Ong WS, Tan SH, et al: Concurrent chemo-radiation with or without induction gemcitabine, Carboplatin, and Paclitaxel: A randomized, phase $2 / 3$ trial in locally advanced nasopharyngeal carcinoma. Int J Radiat Oncol Biol Phys 91: 952-960, 2015.

18. Chan SC, Yeh $\mathrm{CH}$, Yen $\mathrm{TC}, \mathrm{Ng} \mathrm{SH}$, Chang JT, Lin CY, Yen-Ming T, Fan KH, Huang BS, Hsu CL, et al: Clinical utility of simultaneous whole-body 18F-FDG PET/MRI as a single-step imaging modality in the staging of primary nasopharyngeal carcinoma. Eur J Nucl Med Mol Imaging 45: 1297-1308, 2018.

19. Agarwal V, Bell GW, Nam JW and Bartel DP: Predicting effective microRNA target sites in mammalian mRNAs. eLife 4: e05005, 2015.

20. Spence T, Bruce J, Yip KW and Liu FF: MicroRNAs in nasopharyngeal carcinoma. Linchuang Zhongliuxue Zazhi 5: 17, 2016 (In Chinese).

21. Lee KT, Tan JK, Lam AK and Gan SY: MicroRNAs serving as potential biomarkers and therapeutic targets in nasopharyngeal carcinoma: A critical review. Crit Rev Oncol Hematol 103: 1-9, 2016.

22. Chen WY, Liu SY, Chang YS, Yin JJ, Yeh HL, Mouhieddine TH, Hadadeh O, Abou-Kheir W and Liu YN: MicroRNA-34a regulates WNT/TCF7 signaling and inhibits bone metastasis in Ras-activated prostate cancer. Oncotarget 6: 441-457, 2015.

23. Toraih EA, Ibrahiem AT, Fawzy MS, Hussein MH, Al-Qahtani SAM and Shaalan AAM: MicroRNA-34a: A key regulator in the hallmarks of renal cell carcinoma. Oxid Med Cell Longev 2017: 3269379, 2017.

24. Huang G, Du MY, Zhu H, Zhang N, Lu ZW, Qian LX, Zhang W, Tian X, He X and Yin L: miRNA-34a reversed TGF- $\beta$-induced epithelial-mesenchymal transition via suppression of SMAD4 in NPC cells. Biomed Pharmacother 106: 217-224, 2018.

25. Long Z, Wang B, Tao D, Huang Y and Tao Z: Hypofractionated radiotherapy induces miR-34a expression and enhances apoptosis in human nasopharyngeal carcinoma cells. Int J Mol Med 34: $1388-1394,2014$.

26. Cui M, Xiao H, Li Y, Dong J, Luo D, Li H, Feng G, Wang H and Fan S: Total abdominal irradiation exposure impairs cognitive function involving miR-34a-5p/BDNF axis. Biochim Biophys Acta Mol Basis Dis 1863: 2333-2341, 2017.

27. Qiu Y, Guo Z, Han L, Yang Y, Li J, Liu S and Lv X: Network-level dysconnectivity in patients with nasopharyngeal carcinoma (NPC) early post-radiotherapy: Longitudinal resting state fMRI study. Brain Imaging Behav 12: 1279-1289, 2018.

This work is licensed under a Creative Commons Attribution-NonCommercial-NoDerivatives 4.0 International (CC BY-NC-ND 4.0) License. 